\section{Response to: 'Does the risk of lymphoma in patients with RA treated with TNF inhibitors differ according to the histological subtype and the type of TNF inhibitor?' by Nocturne et al}

We thank Nocturne et $a l^{1}$ for their interest and comments on our recent paper, which looked at lymphoma risk over the medium term in a cohort of patients receiving tumour necrosis factor inhibitor (TNFi). ${ }^{1}$ Overall we found no statistically significant difference in the risk compared with conventional standard disease-modifying antirheumatic drugs (csDMARDs) either for all TNFi exposures combined or when each TNFi was analysed separately in comparison to csDMARDs, using a number of different models to categorise treatment exposure.

We agree that lymphoma is a heterogeneous group of malignancies and that the spectrum of lymphomas seen in people with rheumatoid arthritis (RA) is different from that in the general population, with an over-representation of inflammation-driven diffuse large B-cell lymphoma (DLBCL). Therefore, understanding whether TNFi treatment is associated with an increase in the risk of this particular lymphoma subtype is of significant interest, as is whether there is any difference in the risk associated with types of TNFi (soluble receptors or monoclonal antibodies).

In our publication, we did look at the relative risk of DLBCL across all TNFi combined and did not identify a significantly increased risk (propensity decile adjusted HR 1.54 (95\% CI 0.60 to 3.95$)$ ). Undertaking a further analysis stratified by specific TNFi within the British Society for Rheumatology Biologics Register for Rheumatoid Arthritis (BSRBR-RA) dataset, however, would be problematic for the following reasons. First, the power to detect a clinically important difference in risk is limited when stratifying both by lymphoma subtype and drug. Second, a large proportion (>50\%) of participants in the BSRBR-RA who started a first TNFi subsequently switched to an alternative agent, markedly reducing power if the analysis was limited to the first TNFi only.

Nocturne et al also highlight a difference between these data and an adjusted HR presented in a 2013 analysis of the BSRBR-RA dataset by researchers from Cardiff University, specific to etanercept. ${ }^{2}$ As alluded to by Nocturne et al, differences in findings between analysis may reflect a different methodological approach. They highlight the challenges of comparing treatment effects using observational data sources, especially when significant channelling bias exists when treatments are reserved for patients with more severe disease. This channelling will be based not only on disease activity but also on other disease and patient-related factors. These differences between patients should be accounted for within the statistical analysis. The 2013 analysis of lymphoproliferative malignancies limited to patients receiving etanercept was only adjusted for age, whereas our 2016 analysis adjusted for a large number of a priori selected potential baseline confounders, using propensity scores. As an additional point, Baecklund $e t a l^{3}$ studied disease activity over the entire RA disease course when studying the relationship between lymphoma/DLBCL and disease activity. Although a wide range of confounders was considered in our analysis, the BSRBR-RA datasets contain only very limited data on lifelong inflammatory burden, capturing only a snapshot of disease activity at the start of and following TNFi exposure and therefore cannot account for any additional risk from chronic inflammation prior to treatment, which may vary by treatment exposure.

Importantly, neither publication identified an overall increased risk of lymphoma, which has remained a leading concern among regulators, industry, healthcare professionals and patients with respect to TNFi for RA. The BSRBR-RA was powered originally to detect a doubling in the risk of non-Hodgkin's lymphoma. Despite the large sample size of the BSRBR-RA, it is still not powered to detect lesser risks of lymphoma or to study the risk of individual lymphoma subtypes. This highlights the potential for future collaborative research across registries to try and address these outstanding questions in relation to treatment safety. The risk of lymphoma and its subtypes is an identified priority of the European League Against Rheumatism Registers and Observational Drugs Studies Group and work in this area continues. ${ }^{4}$

Louise K Mercer, ${ }^{1}$ James B Galloway, ${ }^{2}$ Mark Lunt, ${ }_{1}^{1}$ Rebecca Davies, ${ }^{1}$ Audrey AL Low, ${ }^{1}$ Will G Dixon, ${ }^{1,2}$ Kath D Watson, ${ }^{1}$

Deborah PM Symmons, ${ }^{1,2}$ Kimme L Hyrich ${ }^{1,2}$

${ }^{1}$ Arthritis Research UK Epidemiology Unit, The University of Manchester, Manchester UK

${ }^{2}$ King's College London, London, UK

Correspondence to Professor Kimme L Hyrich, University of Manchester, Stopford Building, Oxford Road, Manchester M13 9PT, UK; kimme.hyrich@manchester.ac.uk

Twitter Follow William Dixon at @WGDixon

Contributors All authors were involved in the writing and agreed final version of this correspondence.

Competing interests KLH has received honoraria from AbbVie and Pfizer. Provenance and peer review Commissioned; internally peer reviewed.

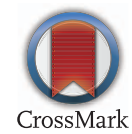

To cite Mercer LK, Galloway JB, Lunt M, et al. Ann Rheum Dis 2017;76:e4.

Received 3 October 2016

Accepted 11 November 2016

Published Online First 1 December 2016

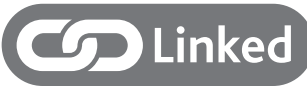

- http://dx.doi.org/10.1136/annrheumdis-2016-210566

Ann Rheum Dis 2017;76:e4. doi:10.1136/annrheumdis-2016-210631

\section{REFERENCES}

1 Nocturne $G$, Seror $R$, Mariette $X$. Does the risk of lymphoma in patients with RA treated with TNF inhibitors differ according to the histological subtype and the type of TNF inhibitor? Ann Rheum Dis 2017;76:e3.

2 Mercer LK, Galloway JB, Lunt M, et al. Risk of lymphoma in patients exposed to antitumour necrosis factor therapy: results from the British Society for Rheumatology Biologics Register for Rheumatoid Arthritis. Ann Rheum Dis Published Online First 8 Aug 2016. doi:10.1136/annrheumdis-2016-209389

3 Morgan CL, Emery P, Porter D, et al. Treatment of rheumatoid arthritis with etanercept with reference to disease-modifying anti-rheumatic drugs: long-term safety and survival using prospective, observational data. Rheumatology (Oxford) 2014;53:186-94.

4 Baecklund E, lliadou A, Askling J, et al. Association of chronic inflammation, not its treatment, with increased lymphoma risk in rheumatoid arthritis. Arthritis Rheum 2006:54:692-701.

5 Mercer L, Mariette $X$, Dixon W, et al. First results of a European registries collaborative project to compare the spectrum of lymphomas between different exposure groups in rheumatoid arthritis. Arthritis Rheum 2014;66:S806-7. 